\title{
SOLUTE TRANSPORT IN POROUS MEDIA. MEDIA WITH CAPILLARIES AS VOIDS*
}

\author{
GUILLERMO H. GOLDSZTEIN ${ }^{\dagger}$
}

\begin{abstract}
We study solute transport in porous media with periodic microstructures consisting of interconnected thin channels. We discuss a local physical mechanism that occurs at the intersections of channels and promotes mixing of the solute with the solvent (i.e., the host liquid). We identify the parameter regime, where this mechanism is the dominant cause of dispersion, and obtain the effective or macroscopic transport equation that the concentration of solute satisfies when the medium is subjected to a time periodic applied pressure gradient. We conclude with illustrative examples.
\end{abstract}

Key words. porous media, solute transport, hydrodynamic dispersion, network approximation, macroscopic behavior, homogenization

AMS subject classifications. 76S05, 76M45, 76M50, 76R05, 76R50

DOI. $10.1137 / 070695228$

1. Introduction. A porous medium is a material that contains relatively small spaces filled with fluid (such as a gas, a liquid, or a mixture of different fluids) embedded in a solid matrix. These fluid filled spaces are called pores or voids. With the exception of metals, some dense rocks, and some plastics, virtually all solid materials are porous to varying degrees.

Solutes are materials that dissolve in liquids forming solutions. An example is salt (not at very large concentrations) in water. The host liquid, such as water in the mentioned example, is called the solvent. The transport of a solute in porous media depends on several factors, including the solvent and solute properties, the fluid velocity field within the porous medium, and the microgeometry, i.e., shape, size, and location of the solid part of the medium and the voids. The objective of this paper is to provide new tools for the study of the influence of these factors on solute transport.

Solute transport in liquid filled porous media plays a significant role in several phenomena of scientific and technological importance including the transport of contaminants in soils [17, 32], the transport of nutrients in bones [50,45, 43, 44, 65, 39], the intrusion of salt in fresh water in soils near ocean coasts, movement of minerals (e.g., fertilizers) in soils, secondary recovery techniques in oil reservoirs (where the injected fluid dissolves the reservoir's oil), the use of tracers in petroleum engineering and hydrology research projects, etc. (see more about these and other examples in $[14,6,9,19,30,58])$.

Several theoretical methods are used to study solute transport in porous media [23]. These include the use of numerical experiments on networks of channels with varying widths forming regular grids $[2,15,16,20,27,58,59]$, percolation methods $[2,7,8,10,47,55,56,57,59,62]$, numerical experiments on media with fractal geometry $[2,16,59,64]$, assuming periodic media and calculating the effective transport equation by means of the method of moments $[2,11,12,13,14,31]$

\footnotetext{
*Received by the editors June 25, 2007; accepted for publication (in revised form) December 11, 2007; published electronically April 2, 2008. This research was supported by the NSF.

http://www.siam.org/journals/siap/68-5/69522.html

${ }^{\dagger}$ School of Mathematics, Georgia Institute of Technology, Atlanta, GA 30332-0160 (ggold@math. gatech.edu).
} 


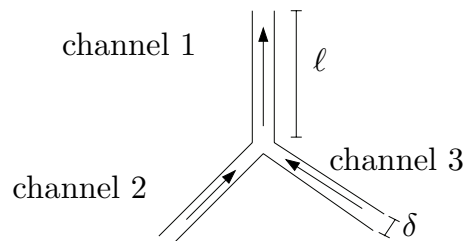

(a)

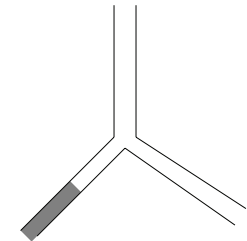

(b)

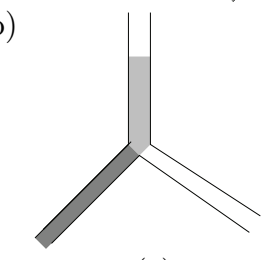

(e)

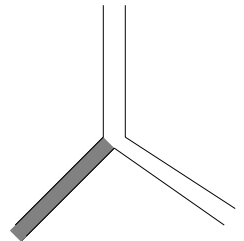

(c)

(d)

FIG. 1.1. (a) Direction of the fluid velocity. (b) Initial distribution of solute concentration. (c) Solute concentration at the time when solute first reaches the intersection. (d) Solute concentration after solute reaches channel 1 if diffusion does not homogenize the solute concentration in slices perpendicular to the channels. (e) Solute concentration after solute reaches channel 1 if the channels are thin enough that diffusion homogenizes the solute concentration in slices perpendicular to the channels. We work in the regime of (e).

or homogenization techniques [42, 48, 49,51], and the calculation of effective transport equations by means of the method of moments on periodic networks of channels $[2,1,29]$ (see also [38] for a study of diffusion in periodic networks with no flow). The most well-known early theoretical works are studies of solute transport in single straight tubes $[63,3]$. We also mention the work on random networks of thin channels $[52,53,54]$, the work on media with trapped fluid in dead-end pores [21], the early work using the method of moments [41], and the work on solute transport in a dilute suspension of spheres [46] and in parallel channels [18]. Further discussions can be found in $[10,36,60,5,24,25,9,26]$. Experimentally, these phenomena have also been extensively studied (see summaries in [37, 30, 22]; see also [40, 28]).

The local phenomenon that motivates our work is simple and described next. Consider the three interconnected channels of Figure 1.1(a). We labeled the channels 1,2 , and 3 . The arrows indicate the direction of the fluid velocity field within each channel. The channels are thin. More precisely, assume that the lengths of the channels are $O(\ell)$, their diameters are $O(\delta)$, the fluid velocities within the channels are $O(v)$, and these parameters satisfy $\delta^{2} / D \ll \ell / v \ll \ell^{2} / D$, where $D$ is the diffusion coefficient of the solute in the solvent under consideration. In this parameter regime, the concentration of solute in each channel is homogeneous in slices perpendicular to the channel and is convected with the average fluid velocity within the channel (see $[63,3]$ and our section 2.1).

In Figure 1.1(b) we display the initial solute concentration, i.e., at time $t=0$. The darker the regions, the larger the solute concentration. Only channel 2 has solute at $t=0$. Let $t_{1}$ be the time when solute first reaches the intersection of the channels. The solute concentration at time $t=t_{1}$ is shown in Figure 1.1(c). Let $t_{2}>t_{1}$. In the absence of diffusion, fluid from channel 2 with solute and fluid from channel 3 without solute would be convected next to each other along channel 1 during the time interval $\left(t_{1}, t_{2}\right)$, and the distribution of solute concentration at $t=t_{2}$ would look as displayed in Figure 1.1(d). This is in contradiction with the fact that solute concentration is homogeneous in slices perpendicular to the channels. In fact, as 
discussed in section 2.1, diffusion homogenizes the concentration of solute in slices perpendicular to the channels, and thus the distribution of solute at $t=t_{2}$ is as illustrated in Figure 1.1(e). During the time interval $\left(t_{1}, t_{2}\right)$, liquid with solute from channel 2 enters channel 1 and mixes with liquid without solute that enters channel 1 from channel 3.

In this paper we consider porous media with periodic microstructures and void spaces consisting of interconnected thin channels. The local effect described in the above paragraph (that corresponds to Figure 1.1(e)) occurs throughout the porous media and promotes solute transport. In this paper we study this phenomenon.

In section 2 we describe our mathematical model. This model is the asymptotic limit of the Navier-Stokes equations within the void with nonslip boundary condition coupled with the convection-diffusion equation for the transport of solute. In section 3 we obtain the macroscopic transport equation which the solute concentration satisfies. We assume that the medium is subjected to a time periodic applied pressure gradient and obtain, by means of homogenization techniques on the model of section 2 , that the solute concentration satisfies a macroscopic convection-diffusion equation. As expected, it is convected with the average fluid velocity. We obtain a relatively simple mean to compute the diffusion tensor, known in the literature as the dispersion tensor. In section 4 we provide some examples and in section 5 conclude with some discussions.

As previously mentioned, there are several methods for studying solute transport in porous media. Each method has its strengths and weaknesses. The most computational economical methods are those that compute the macroscopic properties with the use of periodic networks. This class of methods is essentially limited to [1] and its generalizations [2, 29]. The authors of $[1,2,29]$ use the method of moments instead of homogenization or asymptotic techniques, as we do here. However, this is not the essential difference between those methods and the technique developed in this paper. The models in $[1,2,29]$ use ad hoc rules that correspond to assuming that the volume of the channels is much smaller than the volume of the intersections, and some ad hoc mixing rules are given at the intersections. As a consequence, the physical effect that motivated our work (that of Figure 1.1(e)) is not captured well by the existing models $[1,2,29]$ (see also our section 4). We believe our method is an ideal tool for studying the dependence of the dispersion tensor on the microgeometry and will prove to be very useful.

\section{Mathematical model.}

2.1. Preliminaries. Fluid flow and solute transport in channels. Figure 2.1 shows a two-dimensional channel with length $\ell$ and width $\delta$ filled with a Newtonian incompressible fluid that is subjected to pressures $p=p_{a}$ and $p=p_{b}$ at the ends of the channel. Let $\hat{\mathbf{e}}$ be the vector of unit length parallel to the channel displayed in Figure 2.1. Let $y$ be the coordinate in the direction perpendicular to the channel. At low Reynolds numbers (low velocities), the fluid velocity of the steady state flow is of the form $u(y) \hat{\mathbf{e}}$ with $u$ satisfying $\left(p_{b}-p_{a}\right) / \ell=\mu u^{\prime \prime}$, where $\mu$ is the fluid viscosity and $u^{\prime \prime}$ is the second derivative of $u$. In addition, the fluid velocity satisfies nonslip boundary conditions at the channel walls, i.e., $u=0$ at the walls. Simple calculations show that the velocity has a parabolic profile (see Figure 2.1) and its spatial average across the channel is

$$
\mathbf{v}=\frac{\delta^{2}}{12 \mu \ell}\left(p_{a}-p_{b}\right) \hat{\mathbf{e}}
$$

(see [4]). This type of flow is known as Poiseuille flow. 


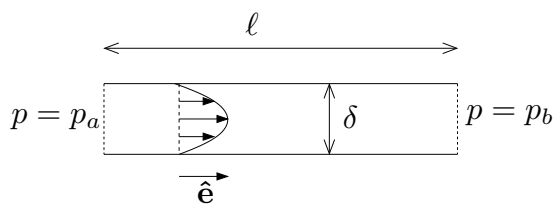

FIG. 2.1. Velocity profile of a Poseuille flow within a straight channel (indicated by arrows).

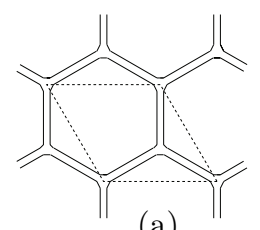

(a)

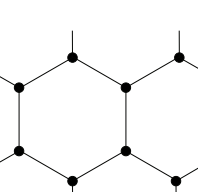

(b)

FIG. 2.2. (a) Example of a periodic network of interconnected channels. The period cell is shown by dashed lines. (b) Associated graph. The lines are the edges and the solid circles the nodes.

Taylor studied solute transport in channels at low Reynolds numbers [63]; see also Aris [3]. The result relevant to us is the following. Let $D$ be the coefficient of diffusion of the solute in the host liquid, $\ell$ the length of the channel, $\delta$ its diameter, and $v$ the spatial average of the norm of the fluid velocity. If

$$
\frac{\delta^{2}}{D} \ll \frac{\ell}{v} \ll \frac{\ell^{2}}{D},
$$

the evolution of solute concentration is described by these two rules:

Rule 1: The concentration of solute is homogeneous in slices (of infinitesimal thickness) perpendicular to the channel.

Rule 2: The solute concentration is convected (or advected) with the average fluid velocity within the channel.

The validity of these two rules can be easily understood as follows. The time required by diffusion to homogenize the solute concentration in slices perpendicular to the channel is of order $O\left(\delta^{2} / D\right)$. Since the time required for solute to be convected from one end to the opposite end of the channel is $O(\ell / v)$, the validity of Rule 1 results from $\delta^{2} / D \ll \ell / v$. On the other hand, Taylor showed that, in the direction of the tube, the time for solute to disperse distances of $O(\ell)$ is $O\left(\ell^{2} / D^{\star}\right)$, where $D^{\star}=O\left(D+v^{2} \delta^{2} / D\right)$. Thus, the validity of Rule 2 results if $\ell / v \ll \ell^{2} / D^{\star}$. Simple algebra shows that, in fact, the two conditions $\delta^{2} / D \ll \ell / v$ and $\ell / v \ll \ell^{2} / D^{\star}$ are equivalent to $(2.2)$.

2.2. Microgeometry. We consider two-dimensional porous media with periodic microstructures. We denote the void or pore space (i.e., the space filled by fluid) by $\Omega_{p}$. Note that $\Omega_{p} \subseteq \mathbb{R}^{2}$. Since the microstructures are periodic, there exist two linearly independent vectors $\mathbf{w}$ and $\mathbf{q}$ such that

$$
\Omega_{p}=\Omega_{p}+n \mathbf{w}+m \mathbf{q}
$$

for all pairs of integers $n$ and $m$. We assume that $\Omega_{p}$ is a collection interconnected thin channels (see Figure 2.2(a)). We assume that exactly three channels merge at each 
intersection. We associate a periodic graph with the microstructure of the medium in a natural way, as illustrated in Figure 2.2(b); the edges are the channels and the nodes the intersection of channels. We denote by $\mathcal{N}$ the set of nodes. We identify the nodes with their location, and thus $\mathcal{N} \subset \mathbb{R}^{2}$. We denote by $\mathcal{E}$ the set of edges. Given an edge $e$, its width (i.e., the width of the channel that corresponds to $e$ ) is denoted by $\delta_{e}$ and its length by $\ell_{e}$. We assume that the widths of the channels are much smaller than their lengths. We also assume that the void space $\Omega_{p}$ is a connected set.

2.3. Fluid flow. Microscopic description. The fluid that fills $\Omega_{p}$ is an incompressible Newtonian fluid with constant density $\rho$ and constant viscosity $\mu$ and satisfies nonslip boundary conditions, i.e., the fluid velocity vanishes at the channels walls (i.e., at the boundary of $\Omega_{p}$ ).

For each node $\mathbf{a} \in \mathcal{N}$, we denote by $p_{\mathbf{a}}$ the pressure at $\mathbf{a}$. Note that $p_{\mathbf{a}}=p_{\mathbf{a}}(t)$ is a function of time $t$. We assume that the medium is subjected to an applied pressure gradient $\mathbf{G}=\mathbf{G}(t)$ that is periodic in $t$ with period $t_{0}$. Thus, the pressures at the nodes satisfy the condition

$$
p_{\mathbf{a}+n \mathbf{w}+m \mathbf{q}}=p_{\mathbf{a}}+\mathbf{G} \cdot(n \mathbf{w}+m \mathbf{q})
$$

for all integers $n$ and $m$ and all nodes a, where, as described above, w and $\mathbf{q}$ are the vectors that determine the periodicity of the microgeometry, and we use the notation $\mathbf{r} \cdot \mathbf{s}=r_{1} s_{1}+r_{2} s_{2}$ for all vectors $\mathbf{r}, \mathbf{s}$, and $r_{i}$ is the ith component of the vector $\mathbf{r}$.

If $e$ is an edge, we denote by $\mathbf{v}_{e}$ the average of the velocity field within the channel $e$. We assume that the variation of $\mathbf{G}(t)$ in time is slow enough that the pressure difference between the two ends of a channel creates a Poiseuille flow within that channel, and thus, for each edge $e$, according to our review (equation (2.1)), we have

$$
\mathbf{v}_{e}=-\frac{\delta_{e}^{2}}{12 \mu \ell_{e}}\left(p_{\mathbf{b}}-p_{\mathbf{a}}\right) \frac{\mathbf{b}-\mathbf{a}}{\|\mathbf{b}-\mathbf{a}\|}, \text { where } \mathbf{a} \text { and } \mathbf{b} \text { are the endpoints of } e
$$

and we use the standard notation for the Euclidean norm $\|\mathbf{r}\|=\sqrt{r_{1}^{2}+r_{2}^{2}}$.

The rate at which the volume of fluid enters an intersection is equal to the rate at which it leaves the intersection, i.e., conservation of mass. This implies that, for each node $\mathbf{a}$, we have

$\sum_{\{e \in \mathcal{E}: \mathbf{a} \text { is an endpoint of } e\}} \delta_{e} \mathbf{v}_{e} \cdot \frac{\mathbf{b}-\mathbf{a}}{\|\mathbf{b}-\mathbf{a}\|}=0$, where $\mathbf{b}$ is the endpoint of $e$ not equal to $\mathbf{a}$.

The velocities within all the channels are uniquely determined by the system (2.5)(2.7). This well-known system (similar models were used as early as [33, 34, 35]; see also $[61,30]$ ) is the asymptotics of the Navier-Stokes equations within the void with nonslip boundary conditions in the limit when the widths of the channels are much smaller than their lengths, and the time variations of the applied pressure gradient $\mathbf{G}(t)$ are slow enough. Note that the resultant velocity field is periodic in space with the same period as the microstructure.

In practice, we first solve for the pressure at the nodes and then for the velocities within the channels. More precisely, using the expression for the velocities in (2.6), we reduce (2.7) into

$$
\sum_{\{\mathbf{b} \in \mathcal{N}: \mathbf{b} \text { is connected to a by an edge }\}} \frac{\delta_{e}^{3}}{12 \mu \ell_{e}}\left(p_{\mathbf{b}}-p_{\mathbf{a}}\right)=0 \text { for all } \mathbf{a} \in \mathcal{N},
$$


which together with condition (2.5) reduce, for each fixed $t$, to a system of linear equations, where the number of unknowns is equal to the number of nodes in a single period cell minus one. Once the pressure at the nodes is obtained, the velocities in the edges are easily computed with (2.6).

2.4. Solute transport. Microscopic description. For each $e \in \mathcal{E}$ we use the notation $v_{e}=\left\|\mathbf{v}_{e}\right\|$. We assume that

$$
v_{e}=O(v), \ell_{e}=O(\ell), \delta_{e}=O(\delta) \text { for all } e \in \mathcal{E},
$$

where $v, \ell$, and $\delta$ are parameters that satisfy (2.2), and thus the transport of solute concentration within each channel is given by Rules 1 and 2 (see (2.3)).

Given an edge $e$, its endpoint with smallest pressure will be called its head and will be denoted by $\mathbf{h}(e)$. Analogously, $\mathbf{k}(e)$, the tail of the edge $e$, is the endpoint of $e$ with largest pressure. Thus, fluid within an edge $e$ (or channel) flows from its tail $\mathbf{k}(e)$ to its head $\mathbf{h}(e)$. Note that, since the fluid flow is time dependent, an endpoint of an edge may be its head for some period of time and its tail for other times.

We parametrize each edge $e$ (more precisely, the segment joining the tail and head of $e$ ) by

$$
\mathbf{x}_{e}(s)=\mathbf{k}(e)+s \frac{\mathbf{h}(e)-\mathbf{k}(e)}{\|\mathbf{h}(e)-\mathbf{k}(e)\|}
$$

and we denote by $u_{e}(s, t)$ the solute concentration in the channel $e$ at the point $\mathbf{x}_{e}(s)$ and time $t$. Note that $\mathbf{x}_{e}(0)=\mathbf{k}(e)$ and $\mathbf{x}_{e}\left(\ell_{e}\right)=\mathbf{h}(e)$ because $\ell_{e}=\|\mathbf{h}(e)-\mathbf{k}(e)\|$. Thus, the channel is parametrized by $\mathbf{x}_{e}(s)$ with $0 \leq s \leq \ell_{e}$. The fact that solute concentration in a channel is convected with the average fluid velocity within the channel translates into

$$
\frac{\partial u_{e}}{\partial t}+v_{e} \frac{\partial u_{e}}{\partial s}=0 \text { for } 0 \leq s \leq \ell_{e}, t \geq 0, \text { and all } e \in \mathcal{E} .
$$

Let $e$ be an edge and $\mathbf{k}(e)$ its tail (at a fixed time $t$ ). One of two cases is possible: $\mathbf{k}(e)$ is the head of two other edges, or $\mathbf{k}(e)$ is the head of only one other edge. Assume first that $\mathbf{k}(e)$ is the head of two other edges, say, $\beta_{1}$ and $\beta_{2}$, i.e., $\mathbf{h}\left(\beta_{1}\right)=\mathbf{h}\left(\beta_{2}\right)=\mathbf{k}(e)$. Conservation of solute implies that solute enters $\mathbf{k}(e)$ at the same rate that it leaves $\mathbf{k}(e)$, and thus $\delta_{e} v_{e} u_{e}(0, t)=\delta_{\beta_{1}} v_{\beta_{1}} u_{\beta_{1}}\left(\ell_{\beta_{1}}, t\right)+\delta_{\beta_{2}} v_{\beta_{2}} u_{\beta_{2}}\left(\ell_{\beta_{2}}, t\right)$. This condition can be written as

$$
u_{e}(0, t)=\frac{\sum_{\{\beta: \mathbf{h}(\beta)=\mathbf{k}(e)\}} \delta_{\beta} v_{\beta} u_{\beta}\left(\ell_{\beta}, t\right)}{\sum_{\{\beta: \mathbf{h}(\beta)=\mathbf{k}(e)\}} \delta_{\beta} v_{\beta}}
$$

once we note that (2.7) at $\mathbf{k}(e)$ is $\delta_{e} v_{e}=\delta_{\beta_{1}} v_{\beta_{1}}+\delta_{\beta_{2}} v_{\beta_{2}}$. We have just shown that (2.12) is valid for edges $e$ for which its tail $\mathbf{k}(e)$ is the head of two other edges. We next show that, in fact, (2.12) is valid for all edges $e$. To that end, assume now that $\mathbf{k}(e)$ is the head of only one edge, say $\beta$. In other words, fluid flows into $\mathbf{k}(e)$ from only channel $\beta$. Thus, the concentration of solute going into $e$ should be equal to the concentration of solute entering $\mathbf{k}(e)$ from $\beta$, i.e., $u_{e}(0, t)=u_{\beta}\left(\ell_{\beta}, t\right)$. This condition is, in fact, (2.12) in this case, i.e., when $\mathbf{k}(e)$ is the head of only one edge.

The system (2.11)-(2.12) uniquely determines the time evolution of the solute concentration within the channels once initial conditions and appropriate boundary conditions are given. We mention that the system (2.11)-(2.12) is not ad hoc; it is the asymptotic limit of the convection-diffusion equation for the transport of solute within the network in the parameter regime in which we work (i.e., (2.9) and (2.2)). 
3. Macroscopic transport equation. We say that two edges are equivalent if one is the translation of the other by a vector of the form $n \mathbf{w}+m \mathbf{q}$, where $\mathbf{w}$ and $\mathbf{q}$ are the vectors that determine the periodicity of the microstructure (see (2.4)) and $n$ and $m$ are integers. Thus, two edges $e_{1}$ and $e_{2}$ are equivalent if there exist $n$ and $m$ integers such that $\mathbf{h}\left(e_{2}\right)=\mathbf{h}\left(e_{1}\right)+n \mathbf{w}+m \mathbf{q}$ and $\mathbf{k}\left(e_{2}\right)=\mathbf{k}\left(e_{1}\right)+n \mathbf{w}+m \mathbf{q}$ (we recall that $\mathbf{h}(e)$ denotes the head of the edge $e$ and $\mathbf{k}(e)$ denotes its tail). This defines an equivalence relation in the set of edges. Note that the widths, lengths, and velocities of equivalent edges are equal, i.e., $\delta_{e_{1}}=\delta_{e_{2}}, \ell_{e_{1}}=\ell_{e_{2}}$, and $\mathbf{v}_{e_{1}}=\mathbf{v}_{e_{2}}$ if $e_{1}$ and $e_{2}$ are equivalent. In what follows we will take spatial average of quantities. Thus, we need to be able to select exactly one edge per equivalence class. We denote by $\mathcal{F}$ a set of edges that contains exactly one edge per equivalent class. For example, $\mathcal{F}$ could be all the edges whose heads are in the period cell

$$
Q=\{s \mathbf{w}+r \mathbf{q}: 0 \leq s, r<1\}
$$

at a certain time.

We first observe that the area occupied by fluid within the period cell $Q$ (i.e., the area of $\left.\Omega_{p} \cap Q\right)$ is

$$
\left|\Omega_{p} \cap Q\right|=\sum_{e \in \mathcal{F}} \delta_{e} \ell_{e}
$$

We denote by $\mathbf{V}$ the spatial average fluid velocity, i.e.,

$$
\mathbf{V}=\frac{\sum_{e \in \mathcal{F}} \delta_{e} \ell_{e} \mathbf{v}_{e}}{\sum_{e \in \mathcal{F}} \delta_{e} \ell_{e}}=\frac{\sum_{e \in \mathcal{F}} \delta_{e} \ell_{e} \mathbf{v}_{e}}{\left|\Omega_{p} \cap Q\right|}
$$

Note that assumption (2.9) implies that $\|\mathbf{V}\|=O(v)$. Assume that $t_{0}$, the period of the applied pressure gradient $\mathbf{G}$, satisfies

$$
t_{0} \gg \frac{\ell}{v} \quad \text { (more precisely } t_{0} \gg \max \ell_{e} / v_{e} \text { most of the time); }
$$

i.e., the time required for solute concentration to be convected across a channel is much smaller than the period of the applied pressure gradient. In Appendix A we show that, macroscopically, the solute concentration is convected with the average fluid velocity $\mathbf{V}$ and dispersed with dispersion tensor

$$
D_{i j}^{\mathrm{eff}}=\frac{1}{t_{0}} \int_{0}^{t_{0}} D_{i j}^{\star}(t) \mathrm{d} t
$$

where

$$
\begin{aligned}
D_{i j}^{\star}=\frac{1}{2\left|\Omega_{p} \cap Q\right|}\{ & \sum_{e \in \mathcal{F}} \delta_{e} \ell_{e}\left(\frac{\ell_{e}}{v_{e}}\left[\mathbf{v}_{e}-\mathbf{V}\right]_{i}\left[\mathbf{v}_{e}-\mathbf{V}\right]_{j}\right. \\
& \left.\left.+\left[\mathbf{V}-\mathbf{v}_{e}\right]_{i}\left[\mathbf{f}_{\mathbf{k}(e)}\right]_{j}+\left[\mathbf{V}-\mathbf{v}_{e}\right]_{j}\left[\mathbf{f}_{\mathbf{k}(e)}\right]_{i}\right)\right\}
\end{aligned}
$$

$[\mathbf{y}]_{i}$ denotes the $i$ th component of the vector $\mathbf{y}$, and the family of vectors $\left(\mathbf{f}_{\mathbf{a}}\right)_{\mathbf{a} \in \mathcal{N}}$ is a solution periodic in space and time (i.e., $\mathbf{f}_{\mathbf{a}}(t)=\mathbf{f}_{\mathbf{a}+n \mathbf{w}+m \mathbf{q}}\left(t+p t_{0}\right)$ for all integers $n, m$, and $p$ ) of the following system:

$$
\sum_{\{e: \mathbf{h}(e)=\mathbf{a}\}} \delta_{e} v_{e}\left(\mathbf{f}_{\mathbf{k}(e)}-\mathbf{f}_{\mathbf{a}}\right)=\sum_{\{e: \mathbf{h}(e)=\mathbf{a}\}} \delta_{e} \ell_{e}\left(\mathbf{v}_{e}-\mathbf{V}\right) \text { for all } \mathbf{a} \in \mathcal{N} .
$$


More precisely, for each $\mathbf{a} \in \mathcal{N}$, let $u_{\mathbf{a}}(t)$ be the solute concentration that leaves the intersection a at time $t$, i.e.,

$$
u_{\mathbf{a}}(t)=u_{e}(0, t) \text { if } \mathbf{a}=\mathbf{k}(e) \text { at time } t .
$$

Note that $u_{\mathbf{a}}(t)$ is well defined because $u_{e_{1}}(0, t)=u_{e_{2}}(0, t)$ if $e_{1}$ and $e_{2}$ are two edges that have the same tail at time $t$, i.e., $\mathbf{k}\left(e_{1}\right)=\mathbf{k}\left(e_{2}\right)$. In Appendix A we show that

$$
u_{\mathbf{a}}(t) \simeq u(\mathbf{a}, t) \text { for } t=O\left(t_{0}^{2} \frac{v}{\ell}\right)
$$

where $u(\mathbf{x}, t)$ satisfies

$$
\frac{\partial u}{\partial t}+\mathbf{V} \cdot \nabla u=\sum_{i, j} D_{i j}^{\mathrm{eff}} \frac{\partial^{2} u}{\partial x_{i} \partial x_{j}},
$$

where $\nabla u$ is the gradient of $u$ with respect to $\mathbf{x}$ and $u$ is subjected to appropriate boundary and initial conditions that depend on the particular problem under consideration. We note that $\mathbf{D}^{\text {eff }}$ is usually referred to as the dispersion tensor.

\section{Examples and observations.}

4.1. Constant applied pressure gradient. As a first general example, we consider the case when the applied pressure gradient $\mathbf{G}$ is time independent. In this case, the system for the pressure at the nodes (2.5) and (2.8) is time independent and so are the velocities within the channels (see (2.6)). The spatially periodic family of vectors $\left(\mathbf{f}_{\mathbf{a}}\right)_{\mathbf{a} \in \mathcal{N}}$, solution of system (3.7), is also time independent, and the expression for the dispersion tensor simplifies to

$$
\text { (4.1) } \begin{aligned}
D_{i j}^{\mathrm{eff}}=D_{i j}^{\star}=\frac{1}{2 \sum_{e \in \mathcal{F}} \delta_{e} \ell_{e}} \sum_{e \in \mathcal{F}} \delta_{e} \ell_{e}( & \frac{\ell_{e}}{v_{e}}\left[\mathbf{v}_{e}-\mathbf{V}\right]_{i}\left[\mathbf{v}_{e}-\mathbf{V}\right]_{j} \\
& \left.+\left[\mathbf{V}-\mathbf{V}_{e}\right]_{i}\left[\mathbf{f}_{\mathbf{k}(e)}\right]_{j}+\left[\mathbf{V}-\mathbf{v}_{e}\right]_{j}\left[\mathbf{f}_{\mathbf{k}(e)}\right]_{i}\right) .
\end{aligned}
$$

4.2. Applied pressure gradient of the form $\mathrm{G}(t)=g(t) \mathrm{E}$ with $\mathrm{E}$ constant. As a second general example, we consider the case when the applied pressure gradient $\mathbf{G}$ is of the form $\mathbf{G}(t)=g(t) \mathbf{E}$ with $\mathbf{E}$ constant and $g(t)$ a real valued periodic function with period $t_{0}$. The evaluation of the dispersion tensor is also simple in this case. Let $\mathbf{D}_{\mathbf{E}}^{\text {eff }}$ be the dispersion tensor that corresponds to the applied pressure gradient $\mathbf{E}$. Then, the dispersion tensor that corresponds to the applied pressure gradient $\mathbf{G}(t)=g(t) \mathbf{E}$ is

$$
\mathbf{D}^{\mathrm{eff}}=\mathbf{D}_{\mathbf{E}}^{\mathrm{eff}} \frac{1}{t_{0}} \int_{0}^{t_{0}}|g(t)| \mathrm{d} t
$$

The validity of the above equation results from simple calculation. Briefly, we first note that, if $\mathbf{v}_{e}^{\mathbf{E}}$ are the velocities within the channels when the applied pressure gradient is $\mathbf{E}$, then $\mathbf{v}_{e}=g(t) \mathbf{v}_{e}^{\mathbf{E}}$ are the velocities within the channels when the applied pressure gradient is $g(t) \mathbf{E}$. As a consequence, if the vectors $\mathbf{f}_{a}^{\mathbf{E}}$ solve system (3.7) when the applied pressure gradient is $\mathbf{E}$, then $\mathbf{f}_{a}=g(t) \mathbf{f}_{a}^{\mathbf{E}}$ is a solution of system (3.7) when the applied pressure gradient is $g(t) \mathbf{E}$. Thus, if $\mathbf{D}_{\mathbf{E}}^{\star}$ is the tensor of (3.6) when the applied pressure gradient is $\mathbf{E}$, then $\mathbf{D}^{\star}=|g(t)| \mathbf{D}_{\mathbf{E}}^{\star}$ is the tensor of (3.6) when the applied pressure gradient is $g(t) \mathbf{E}$. Note that $\mathbf{D}_{\mathbf{E}}^{\star}$ is time independent, and thus $\mathbf{D}_{\mathbf{E}}^{\text {eff }}=\mathbf{D}_{\mathbf{E}}^{\star}$ (see (3.6)). Finally, (4.2) results from (3.6). 


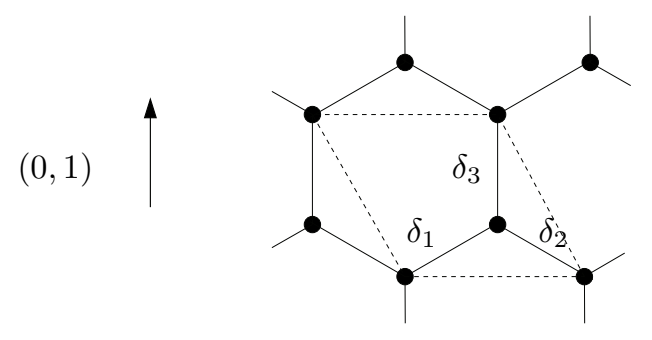

FIG. 4.1. Graph corresponding to the microgeometry of our example. The period cell is enclosed by dashed lines. The widths of the channels are $\delta_{1}, \delta_{2}$, and $\delta_{3}$.

4.3. A concrete example. The graph that corresponds to the microgeometry of our example is shown in Figure 4.1. All the channels have the same length $\ell$ and form regular hexagons. The period cell is enclosed by dashed lines. The width of the channels in the period cell are, as displayed in the figure, $\delta_{1}, \delta_{2}$, and $\delta_{3}$. We assume the applied pressure gradient to be of the form

$$
\mathbf{G}(\mathbf{t})=\left(0, \frac{g(t)}{\ell}\right)
$$

where $g$ is a periodic function with period $t_{0}$ and $(0,1)$ is the unit vector that points in the vertical direction (see Figure 4.1). Some algebra shows that, in this example, the use of our method leads to

$$
\mathbf{V}_{2}(t)=-\frac{3}{16 \mu \ell} \frac{\delta_{3}^{3}\left(\delta_{1}^{3}+\delta_{2}^{3}\right)}{\left(\delta_{1}^{3}+\delta_{2}^{3}+\delta_{3}^{3}\right)\left(\delta_{1}+\delta_{2}+\delta_{3}\right)} g(t)
$$

and

$$
\mathbf{D}_{22}^{\mathrm{eff}}=\frac{9}{64 \mu} \frac{\delta_{3}^{3}\left(\delta_{1}^{3}+\delta_{2}^{3}\right)\left(\delta_{1}+\delta_{2}\right)^{2}\left(\delta_{1}-\delta_{2}\right)^{2}}{\delta_{1} \delta_{2}\left(\delta_{1}^{3}+\delta_{2}^{3}+\delta_{3}^{3}\right)\left(\delta_{1}+\delta_{2}+\delta_{3}\right)^{3}} \frac{1}{t_{0}} \int_{0}^{t_{0}}|g(t)| \mathrm{d} t .
$$

To discuss the above formulas in a more concrete context, assume that the material occupies the region $x_{2}>0$. Also assume that the material is attached to a reservoir of solute located at $x_{2}<0$ and that initially there is no solute within the material (for $x_{2}>0$ ). Due to symmetry, the solute concentration $u$, solution of (3.10), in this example depends only on $x_{2}$. Thus, we need only $\mathbf{V}_{2}$ and $\mathbf{D}_{22}^{\text {eff }}$, which are given by (4.4) and (4.5), respectively.

As a first observation, note that $\mathbf{D}_{22}^{\mathrm{eff}}=0$ if $\delta_{1}=\delta_{2}$. Thus, after each period, solute is convected a distance $\int_{0}^{t_{0}} \mathbf{V}(t) \mathrm{d} t$ but is not dispersed in our asymptotic limit; there is a smaller order effective dispersion that results from an effect known as Taylor dispersion inside the channels $[63,3]$. Note that this is in accordance with the physical effect described in the introduction as shown in Figure 1.1(e). The mixing of solute with the host liquid occurs when solute from two different channels and at different concentrations flows into the same intersection (in Figure 1.1(e) one of the channels had zero solute concentration). Due to symmetry in our example when $\delta_{1}=\delta_{2}$, whenever solute from two channels flows into the same intersection, the concentration in both channels is the same. This is illustrated in Figure 4.2(a), where we show that solute reaches the upper ends of all the channels attached to the reservoir at the same time if $\delta_{1}=\delta_{2}$. 


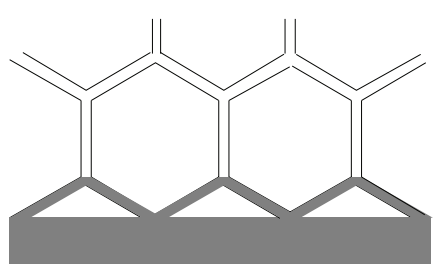

(a)

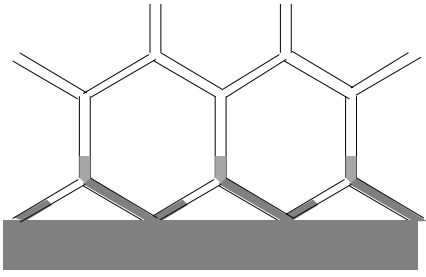

(b)

Fig. 4.2. Microgeometry that corresponds to the graph of Figure 4.1. In (a), $\delta_{1}=\delta_{2}$. In (b), $\delta_{1}<\delta_{2}$. The shaded areas represent the solute concentration. The darker the shade, the larger the solute concentration.

On the other hand, in Figure 4.2(b) we display an example where $\delta_{1}<\delta_{2}$. As illustrated in that figure, the time required for solute from the reservoir to travel through the thinner channels is longer than the travel time through the thicker channels. Thus, the effect illustrated in Figure 1.1(e) does occur and, as (4.5) implies, we have that $D_{22}^{\text {eff }} \neq 0$. Note in particular that, in the case $\int_{0}^{t_{0}} g(t) \mathrm{d} t=0$, there is no convection after a complete period. Thus, there will be much more transport of solute in our second example, where $D_{22}^{\text {eff }} \neq 0$, than in the example of the previous paragraph, where $D_{22}^{\mathrm{eff}}=0$.

At first glance, (4.5) seems to lead to the following contradiction. On one hand, (4.5) shows that $D_{22}^{\text {eff }} \rightarrow \infty$ as $\delta_{1} \rightarrow 0$ while keeping $\delta_{2}$ and $\delta_{3}$ fixed. However, $\delta_{1}=0$ means not having the channels with width $\delta_{1}$, and thus not having intersection of three channels. According to our discussions, we would expect $D_{22}^{\text {eff }}=0$ in this case. This apparent contradiction is resolved by (3.4) and (3.9) which state that (4.5) is valid for $t=O\left(t_{0}^{2} v / \ell\right)$ and $t_{0} \gg \ell / v_{1}$, and thus we need $t \gg \ell / v_{1}$. Finally, we note that, since $v_{1} \rightarrow 0$ as $\delta_{1} \rightarrow 0$, this is a singular limit, which resolves this apparent contradiction. In other words, the smaller $\delta_{1}$, the longer we have to wait for dispersion to occur and for our asymptotics to be valid. As $\delta_{1} \rightarrow 0$, we would have to wait an infinitely long time.

5. Discussion. As mentioned in the introduction, there are several methods for studying solute transport in porous media. For their computational efficiency and their flexibility in modeling microstructures, methods that compute the macroscopic properties with the use of periodic networks are very useful. So far, this class of methods is essentially limited to [1] and its generalizations [2, 29]. Moreover, as mentioned in the introduction, the models in $[1,2,29]$ use ad hoc rules that prevent them from accurately modeling the physical effect that motivated the present work, i.e., that of Figure 1.1(e).

Thus, while a large body of work exists in solute transport in porous media, the work introduced here is new and, we believe, will prove powerful in providing new understanding of the dependence of solute transport on the microgeometry. The strengths of our method include the following: (1) This method is exact to first order, i.e., has a small error. More precisely, it is the asymptotic limit of the wellestablished Navier-Stokes system for fluid flow and the convection-diffusion equation for solute transport (there are no ad hoc rules imposed). (2) The asymptotic limit used results from considering the simple but, we believe, fundamental effect of Figure 1.1(e). While this local effect has been identified and appears in standard texts on porous media [30], its global consequence (i.e., the combined effect of this phenomenon 
at all intersections) has not been well studied. This method is an ideal tool for those studies. (3) This method is relatively computationally inexpensive, essentially solving a linear system whose number of variables is equal to two times the number of nodes in a period cell. We mention that the extension of our method to three dimension is immediate.

\section{Appendix A. Asymptotic approximation.}

A.1. Dimensionless variables, parameters, and equations. We first define the small dimensionless parameter $\varepsilon$ as

$$
\varepsilon=\frac{\ell}{v t_{0}} \ll 1
$$

for each edge $e$, the dimensionless parameters as

$$
\bar{\ell}_{e}=\frac{\ell_{e}}{\ell} \text { and } \bar{\delta}_{e}=\frac{\delta_{e}}{\delta},
$$

the dimensionless velocities and their norms as

$$
\overline{\mathbf{v}}_{e}=\frac{\mathbf{v}_{e}}{v} \text { and } \bar{v}_{e}=\left\|\overline{\mathbf{v}}_{e}\right\|,
$$

and the dimensionless average velocity as

$$
\overline{\mathbf{V}}=\frac{\mathbf{V}}{v} .
$$

The velocities will be periodic with the same period $t_{0}$ as the applied pressure gradient. This motivates the choice of the dimensionless time

$$
\bar{t}=\frac{t}{t_{0}} .
$$

We regard the dimensionless velocities as 1-periodic functions of the dimensionless time; i.e., $\overline{\mathbf{v}}_{e}=\overline{\mathbf{v}}_{e}(\bar{t})$ and $\overline{\mathbf{V}}=\overline{\mathbf{V}}(\bar{t})$ are periodic with period 1.

Since the velocities are of order $v$, distances traveled by convection in periods of times of order $t_{0}$ are of order $v t_{0}$. This motivates the use of the following space dimensionless variable:

$$
\overline{\mathbf{x}}=\frac{\mathbf{x}}{v t_{0}} .
$$

Accordingly, the dimensionless nodes are the set

$$
\overline{\mathcal{N}}=\frac{\mathcal{N}}{v t_{0}},
$$

the dimensionless head and tail of each edge $e$ are

$$
\overline{\mathbf{h}}(e)=\frac{\mathbf{h}(e)}{v t_{0}} \text { and } \overline{\mathbf{k}}(e)=\frac{\mathbf{k}(e)}{v t_{0}},
$$

respectively, and the dimensionless vectors that determine the periodicity of the microstructure are

$$
\overline{\mathbf{w}}=\frac{\mathbf{w}}{v t_{0}} \text { and } \overline{\mathbf{q}}=\frac{\mathbf{q}}{v t_{0}} \text {. }
$$

Copyright $@$ by SIAM. Unauthorized reproduction of this article is prohibited. 
Thus, parametrizing the segment joining $\overline{\mathbf{k}}(e)$ and $\overline{\mathbf{h}}(e)$ by

$$
\overline{\mathbf{x}}_{e}(\bar{s})=\overline{\mathbf{k}}(e)+\bar{s} \frac{\overline{\mathbf{h}}(e)-\overline{\mathbf{k}}(e)}{\|\overline{\mathbf{h}}(e)-\overline{\mathbf{k}}(e)\|},
$$

we have that (2.11) becomes

$$
\frac{\partial \bar{u}_{e}}{\partial \bar{t}}+\bar{v}_{e} \frac{\partial \bar{u}_{e}}{\partial \bar{s}}=0 \text { for } 0 \leq \bar{s} \leq \varepsilon \bar{\ell}_{e}, \bar{t} \geq 0, \text { and all } e \in \mathcal{E},
$$

where $\bar{u}_{e}(\bar{s}, \bar{t})$ is the solute concentration in the channel $e$ at the point $v t_{0} \overline{\mathbf{x}}_{e}(\bar{s})$ and time $t_{0} \bar{t}$. Note that $\overline{\mathbf{k}}(e)=\overline{\mathbf{x}}_{e}(0)$ and $\overline{\mathbf{h}}(e)=\overline{\mathbf{x}}_{e}\left(\varepsilon \bar{\ell}_{e}\right)$.

On the other hand, (2.12) becomes

$$
\bar{u}_{e}(0, \bar{t})=\frac{\sum_{\{\beta: \overline{\mathbf{h}}(\beta)=\overline{\mathbf{k}}(e)\}} \bar{\delta}_{\beta} \bar{v}_{\beta} \bar{u}_{\beta}\left(\varepsilon \bar{\ell}_{\beta}, \bar{t}\right)}{\sum_{\{\beta: \overline{\mathbf{h}}(\beta)=\overline{\mathbf{k}}(e)\}} \bar{\delta}_{\beta} \bar{v}_{\beta}} .
$$

A.2. Solute transport within each channel. Let $e$ be an edge. Solute is convected from $\overline{\mathbf{k}}(e)$, the tail of $e$, to $\overline{\mathbf{h}}(e)$, the head of $e$. Thus, the solute concentration at $\overline{\mathbf{h}}(e)$ at time $\bar{t}$ is equal to the solute concentration at $\overline{\mathbf{k}}(e)$ at an earlier time $\bar{t}-\Delta \bar{t}_{e}$, i.e.,

$$
\bar{u}_{e}\left(0, \bar{t}-\Delta \bar{t}_{e}\right)=\bar{u}_{e}\left(\varepsilon \bar{\ell}_{e}, \bar{t}\right) .
$$

We next compute $\Delta \bar{t}_{e}$.

Let $S(\tau)$ be the solution of

$$
S^{\prime}(\tau)=\bar{v}_{e}(\tau) \text { and } S(\bar{t})=\varepsilon \bar{\ell}_{e},
$$

where $S^{\prime}$ is the derivative of $S$. We claim that $\Delta \bar{t}_{e}$ is the solution of

$$
S\left(\bar{t}-\Delta \bar{t}_{e}\right)=0 .
$$

This is due to the fact that $\bar{u}_{e}(S(\tau), \tau)$ is independent of $\tau$, and thus $\bar{u}_{e}\left(0, \bar{t}-\Delta \bar{t}_{e}\right)=$ $\bar{u}_{e}\left(S\left(\bar{t}-\Delta \bar{t}_{e}\right), \bar{t}-\Delta \bar{t}_{e}\right)=\bar{u}_{e}(S(\bar{t}), \bar{t})=\bar{u}_{e}\left(\varepsilon \bar{\ell}_{e}, \bar{t}\right)$.

From (A.14) and (A.15), we note that $\Delta \bar{t}_{e}=O(\varepsilon)$. Thus, we Taylor expand (A.15) to get

$$
0=S\left(\bar{t}-\Delta \bar{t}_{e}\right) \simeq S(\bar{t})-S^{\prime}(\bar{t}) \Delta \bar{t}_{e}+\frac{S^{\prime \prime}(\bar{t})}{2}\left(\Delta \bar{t}_{e}\right)^{2} .
$$

Next we note that $S(\bar{t})=\varepsilon \bar{\ell}_{e}$ and $S^{\prime}(\bar{t})=\bar{v}_{e}(\bar{t})$ (see (A.14)). Thus, $S^{\prime \prime}(\bar{t})=\bar{v}_{e}^{\prime}(\bar{t})$, and we conclude from (A.16) that

$$
0 \simeq \varepsilon \bar{\ell}_{e}-\bar{v}_{e}(\bar{t}) \Delta \bar{t}_{e}+\frac{\bar{v}_{e}^{\prime}(\bar{t})}{2}\left(\Delta \bar{t}_{e}\right)^{2} .
$$

We now set $\Delta \bar{t}_{e}=\varepsilon \Delta \bar{t}_{1}+\varepsilon^{2} \Delta \bar{t}_{2}$, plug this expression into (A.17), collect powers of $\varepsilon$ to obtain equations for $\Delta \bar{t}_{1}$ and $\Delta \bar{t}_{2}$, and finally get

$$
\Delta \bar{t}_{e} \simeq \varepsilon \frac{\bar{\ell}_{e}}{\bar{v}_{e}}+\varepsilon^{2} \frac{\bar{\ell}_{e}^{2}}{2} \frac{\bar{v}_{e}^{\prime}}{\bar{v}_{e}^{3}}
$$

where $\bar{v}_{e}$ and $\bar{v}_{e}^{\prime}$ are evaluated at $\bar{t}$. Note that $\Delta \bar{t}_{e}$ is a function of $\bar{t}$. 
A.3. Continuum approximation. Using (A.13), (A.12) becomes

$$
\bar{u}_{e}(0, \bar{t})=\frac{\sum_{\{\beta: \overline{\mathbf{h}}(\beta)=\overline{\mathbf{k}}(e)\}} \bar{\delta}_{\beta} \bar{v}_{\beta} \bar{u}_{\beta}\left(0, \bar{t}-\Delta \bar{t}_{\beta}\right)}{\sum_{\{\beta: \overline{\mathbf{h}}(\beta)=\overline{\mathbf{k}}(e)\}} \bar{\delta}_{\beta} \bar{v}_{\beta}},
$$

where $\Delta \bar{t}_{\beta}$ is given by (A.18).

For each dimensionless node $\overline{\mathbf{a}} \in \overline{\mathcal{N}}$, let $\bar{u}_{\overline{\mathbf{a}}}(\bar{t})$ be the solute concentration that leaves the intersection $\overline{\mathbf{a}}$, i.e.,

$$
\bar{u}_{\overline{\mathbf{a}}}(\bar{t})=\bar{u}_{e}(0, \bar{t}) \text { if } \overline{\mathbf{a}}=\overline{\mathbf{k}}(e) \text { at time } \bar{t} .
$$

With the notation introduced in (A.20), (A.19) becomes

$$
\bar{u}_{\overline{\mathbf{a}}}(\bar{t})=\frac{\sum_{\{\beta: \overline{\mathbf{h}}(\beta)=\overline{\mathbf{a}}\}} \bar{\delta}_{\beta} \bar{v}_{\beta} \bar{u}_{\overline{\mathbf{k}}(\beta)}\left(\bar{t}-\Delta \bar{t}_{\beta}\right)}{\sum_{\{\beta: \overline{\mathbf{h}}(\beta)=\overline{\mathbf{a}}\}} \bar{\delta}_{\beta} \bar{v}_{\beta}} .
$$

A.3.1. Ansatz and expansions. We now use standard asymptotic techniques to obtain the macroscopic transport equation for the solute concentration. We propose the ansatz

$$
\bar{u}_{\overline{\mathbf{a}}}(\bar{t})=\rho(\overline{\mathbf{a}}, \bar{t}, \varepsilon \bar{t})+\varepsilon f_{\hat{\mathbf{a}}}(\overline{\mathbf{a}}, \bar{t}, \varepsilon \bar{t})+\varepsilon^{2} g_{\hat{\mathbf{a}}}(\overline{\mathbf{a}}, \bar{t}, \varepsilon \bar{t}), \text { where } \hat{\mathbf{a}}=\frac{\overline{\mathbf{a}}}{\varepsilon},
$$

$\rho(\overline{\mathbf{x}}, \bar{t}, \tau)$ is a smooth function of its variables periodic in $\bar{t}$ with period 1 , and for each $\hat{\mathbf{a}} \in \overline{\mathcal{N}} / \varepsilon=\mathcal{N} / \ell$ the functions $f_{\hat{\mathbf{a}}}(\overline{\mathbf{x}}, \bar{t}, \tau)$ and $g_{\hat{\mathbf{a}}}(\overline{\mathbf{x}}, \bar{t}, \tau)$ are smooth functions of $\overline{\mathbf{x}}, \bar{t}$, and $\tau$ and are also periodic in $\bar{t}$ with period 1 . The family of functions $f_{\hat{\mathbf{a}}}$ and $g_{\hat{\mathbf{a}}}$ are periodic in $\hat{\mathbf{a}}$ in the sense that $f_{\hat{\mathbf{a}}+(n \overline{\mathbf{w}}+m \overline{\mathbf{q}}) / \varepsilon}(\overline{\mathbf{x}}, \bar{t}, \tau)=f_{\hat{\mathbf{a}}}(\overline{\mathbf{x}}, \bar{t}, \tau)$ and $g_{\hat{\mathbf{a}}+(n \overline{\mathbf{w}}+m \overline{\mathbf{q}}) / \varepsilon}(\overline{\mathbf{x}}, \bar{t}, \tau)=g_{\hat{\mathbf{a}}}(\overline{\mathbf{x}}, \bar{t}, \tau)$ for all integers $n$ and $m$ and $\hat{\mathbf{a}} \in \overline{\mathcal{N}} / \varepsilon$.

Let $\overline{\mathbf{x}}$ be a point that we hold fixed for the moment. Let $\overline{\mathbf{a}}$ be a dimensionless node $\overline{\mathbf{a}} \in \overline{\mathcal{N}}$ such that $\|\overline{\mathbf{x}}-\overline{\mathbf{a}}\|=O(\varepsilon)$. We write

$$
\overline{\mathbf{a}}=\overline{\mathbf{x}}+\varepsilon(\hat{\mathbf{a}}-\hat{\mathbf{x}}), \text { where } \hat{\mathbf{a}}=\frac{\overline{\mathbf{a}}}{\varepsilon} \text { and } \hat{\mathbf{x}}=\frac{\overline{\mathbf{x}}}{\varepsilon} .
$$

We now plug this expression for $\overline{\mathbf{a}}$ into the right-hand side of (A.22) to get

$$
\begin{array}{r}
\bar{u}_{\overline{\mathbf{a}}}(\bar{t})=\rho(\overline{\mathbf{x}}+\varepsilon(\hat{\mathbf{a}}-\hat{\mathbf{x}}), \bar{t}, \varepsilon \bar{t})+\varepsilon f_{\hat{\mathbf{a}}}(\overline{\mathbf{x}}+\varepsilon(\hat{\mathbf{a}}-\hat{\mathbf{x}}), \bar{t}, \varepsilon \bar{t}) \\
+\varepsilon^{2} g_{\hat{\mathbf{a}}}(\overline{\mathbf{x}}+\varepsilon(\hat{\mathbf{a}}-\hat{\mathbf{x}}), \bar{t}, \varepsilon \bar{t}) .
\end{array}
$$

We now Taylor expand the right-hand side of the above equality around the point $(\overline{\mathbf{x}}, \bar{t}, \varepsilon \bar{t})$ to get

$$
\begin{array}{r}
\bar{u}_{\overline{\mathbf{a}}}(\bar{t}) \simeq \rho+\varepsilon\left\{\sum_{i} \frac{\partial \rho}{\partial \bar{x}_{i}}[\hat{\mathbf{a}}-\hat{\mathbf{x}}]_{i}+f_{\hat{\mathbf{a}}}\right\} \\
+\varepsilon^{2}\left\{\frac{1}{2} \sum_{i, j} \frac{\partial^{2} \rho}{\partial \bar{x}_{i} \partial \bar{x}_{j}}[\hat{\mathbf{a}}-\hat{\mathbf{x}}]_{i}[\hat{\mathbf{a}}-\hat{\mathbf{x}}]_{j}+\sum_{i} \frac{\partial f_{\hat{\mathbf{a}}}}{\partial \bar{x}_{i}}[\hat{\mathbf{a}}-\hat{\mathbf{x}}]_{i}+g_{\hat{\mathbf{a}}}\right\},
\end{array}
$$

where $\rho, f_{\hat{\mathbf{a}}}, g_{\hat{\mathbf{a}}}$ and their derivatives are evaluated at $(\overline{\mathbf{x}}, \bar{t}, \varepsilon \bar{t})$. In the above equation we neglected terms of order $\varepsilon^{3}$.

Copyright $\odot$ by SIAM. Unauthorized reproduction of this article is prohibited. 
Now let $\beta$ be an edge whose dimensionless head is $\overline{\mathbf{a}}$, i.e., $\overline{\mathbf{h}}(\beta)=\overline{\mathbf{a}}$. Note that $\|\overline{\mathbf{k}}(\beta)-\overline{\mathbf{a}}\|=\varepsilon \bar{\ell}_{\beta}$. Thus, since $\|\overline{\mathbf{x}}-\overline{\mathbf{a}}\|=O(\varepsilon)$, we have $\|\overline{\mathbf{x}}-\overline{\mathbf{k}}(\beta)\|=O(\varepsilon)$. We write

$$
\overline{\mathbf{k}}(\beta)=\overline{\mathbf{x}}+\varepsilon(\hat{\mathbf{k}}(\beta)-\hat{\mathbf{x}}) \text {, where } \hat{\mathbf{k}}(\beta)=\frac{\overline{\mathbf{k}}(\beta)}{\varepsilon} \text { and } \hat{\mathbf{x}}=\frac{\overline{\mathbf{x}}}{\varepsilon} .
$$

From (A.24), but replacing $\overline{\mathbf{a}}$ by $\overline{\mathbf{k}}(\beta)$ and $\bar{t}$ by $\bar{t}-\Delta \bar{t}_{\beta}$, we get

$$
\begin{aligned}
\bar{u}_{\overline{\mathbf{k}}(\beta)}\left(\bar{t}-\Delta \bar{t}_{\beta}\right)=\rho & \left(\overline{\mathbf{x}}+\varepsilon(\hat{\mathbf{k}}(\beta)-\hat{\mathbf{x}}), \bar{t}-\Delta \bar{t}_{\beta}, \varepsilon \bar{t}-\varepsilon \Delta \bar{t}_{\beta}\right) \\
& +\varepsilon f_{\hat{\mathbf{k}}(\beta)}\left(\overline{\mathbf{x}}+\varepsilon(\hat{\mathbf{k}}(\beta)-\hat{\mathbf{x}}), \bar{t}-\Delta \bar{t}_{\beta}, \varepsilon \bar{t}-\varepsilon \Delta \bar{t}_{\beta}\right) \\
& +\varepsilon^{2} g_{\hat{\mathbf{k}}(\beta)}\left(\overline{\mathbf{x}}+\varepsilon(\hat{\mathbf{k}}(\beta)-\hat{\mathbf{x}}), \bar{t}-\Delta \bar{t}_{\beta}, \varepsilon \bar{t}-\varepsilon \Delta \bar{t}_{\beta}\right) .
\end{aligned}
$$

We now Taylor expand the right-hand side of the above equation around the point $(\overline{\mathbf{x}}, \bar{t}, \varepsilon \bar{t})$ and make use of the expression (A.18) (with $e$ replaced by $\beta$ ) to get

$$
\begin{array}{r}
\bar{u}_{\overline{\mathbf{k}}(\beta)}\left(\bar{t}-\Delta \bar{t}_{\beta}\right) \simeq \rho+\varepsilon\left\{\sum_{i} \frac{\partial \rho}{\partial \bar{x}_{i}}[\hat{\mathbf{k}}(\beta)-\hat{\mathbf{x}}]_{i}-\frac{\partial \rho}{\partial \bar{t}} \frac{\bar{\ell}_{\beta}}{\bar{v}_{\beta}}+f_{\hat{\mathbf{k}}(\beta)}\right\} \\
+\varepsilon^{2}\left\{\frac{1}{2} \sum_{i, j} \frac{\partial^{2} \rho}{\partial \bar{x}_{i} \partial \bar{x}_{j}}[\hat{\mathbf{k}}(\beta)-\hat{\mathbf{x}}]_{i}[\hat{\mathbf{k}}(\beta)-\hat{\mathbf{x}}]_{j}-\sum_{i} \frac{\partial^{2} \rho}{\partial \bar{t} \partial \bar{x}_{i}}[\hat{\mathbf{k}}(\beta)-\hat{\mathbf{x}}]_{i} \frac{\bar{\ell}_{\beta}}{\bar{v}_{\beta}}\right. \\
\left.+\frac{1}{2} \frac{\partial^{2} \rho}{\partial \bar{t}^{2}} \frac{\bar{\ell}_{\beta}^{2}}{\bar{v}_{\beta}^{2}}-\frac{\partial \rho}{\partial \bar{t}} \frac{\bar{\ell}_{\beta}^{2}}{2} \frac{\bar{v}_{\beta}^{\prime}}{\bar{v}_{\beta}^{3}}-\frac{\partial \rho}{\partial \tau} \frac{\bar{\ell}_{\beta}}{\bar{v}_{\beta}}+\sum_{i} \frac{\partial f_{\hat{\mathbf{k}}(\beta)}}{\partial \bar{x}_{i}}[\hat{\mathbf{k}}(\beta)-\hat{\mathbf{x}}]_{i}-\frac{\partial f_{\hat{\mathbf{k}}(\beta)}}{\partial \bar{t}} \frac{\bar{\ell}_{\beta}}{\bar{v}_{\beta}}+g_{\hat{\mathbf{k}}(\beta)}\right\},
\end{array}
$$

where $\rho, f_{\hat{\mathbf{k}}(\beta)}, g_{\hat{\mathbf{k}}(\beta)}$ and their derivatives are evaluated in $(\bar{x}, \bar{t}, \varepsilon \bar{t})$, and $\bar{v}_{\beta}$ and its derivative are evaluated at $\bar{t}$. In the above equation we neglected terms of order $\varepsilon^{3}$.

Now we plug the expressions for $\bar{u}_{\hat{\mathbf{a}}}(\bar{t})$ and $\bar{u}_{\overline{\mathbf{k}}(\beta)}\left(\bar{t}-\Delta \bar{t}_{\beta}\right)$ given in (A.25) and (A.28) into (A.21), neglect terms of $\varepsilon^{2}$, and make simple algebraic manipulations (which include dividing by $\varepsilon$ ) to obtain

$$
\sum_{\beta: \overline{\mathbf{h}}(\beta)=\overline{\mathbf{a}}} \bar{\delta}_{\beta} \bar{v}_{\beta}\left(f_{\hat{\mathbf{k}}(\beta)}-f_{\hat{\mathbf{a}}}\right)=\left(\sum_{\beta: \overline{\mathbf{h}}(\beta)=\overline{\mathbf{a}}} \bar{\delta}_{\beta} \bar{\ell}_{\beta}\right) \frac{\partial \rho}{\partial \bar{t}}+\left(\sum_{\beta: \overline{\mathbf{h}}(\beta)=\overline{\mathbf{a}}} \bar{\delta}_{\beta} \bar{\ell}_{\beta} \overline{\mathbf{v}}_{\beta}\right) \cdot \bar{\nabla} \rho .
$$

We require the above equation to be valid for all $(\bar{x}, \bar{t}, \tau)$, not just at $\tau=\varepsilon \bar{t}$.

A.3.2. Fredholm alternative. First equation for $\rho$. Convection with average velocity. We recall that two edges are equivalent if one is the translation of the other by a vector of the form $n \mathbf{w}+m \mathbf{q}$, where $n$ and $m$ are integers, and we denote by $\mathcal{F}$ a set of edges that contains exactly one edge per equivalent class (see section 3). Analogously, we also say that two dimensionless nodes $\overline{\mathbf{a}}$ and $\overline{\mathbf{b}}$ are equivalent if $\overline{\mathbf{b}}=\overline{\mathbf{a}}+n \overline{\mathbf{w}}+m \overline{\mathbf{q}}$ for some $n$ and $m$ integers, and we denote by $\overline{\mathcal{M}}$ a set of dimensionless nodes that contains exactly one dimensionless node per equivalent class. For example, $\overline{\mathcal{M}}$ could be all the dimensionless nodes included in the dimensionless period cell $\bar{Q}=\{s \overline{\mathbf{w}}+r \overline{\mathbf{q}}: 0 \leq s, r<1\}$, i.e., $\overline{\mathcal{M}}=\overline{\mathcal{N}} \cap \bar{Q}$.

Let $y_{\hat{\mathbf{a}}}$ be defined for all $\hat{\mathbf{a}} \in \overline{\mathcal{N}} / \varepsilon$ and have the same periodicity as $f_{\hat{\mathbf{a}}}$, i.e., $y_{\hat{\mathbf{a}}+(n \overline{\mathbf{w}}+m \overline{\mathbf{q}}) / \varepsilon}=y_{\hat{\mathbf{a}}}$ for all integers $n$ and $m$ and all $\hat{\mathbf{a}} \in \overline{\mathcal{N}} / \varepsilon$. Note that

$$
\sum_{\overline{\mathbf{a}} \in \bar{M}} y_{\hat{\mathbf{a}}} \sum_{\beta: \overline{\mathbf{h}}(\beta)=\overline{\mathbf{a}}} \bar{\delta}_{\beta} \bar{v}_{\beta} f_{\hat{\mathbf{k}}(\beta)}=\sum_{\beta \in \mathcal{F}} \bar{\delta}_{\beta} \bar{v}_{\beta} y_{\hat{\mathbf{h}}(\beta)} f_{\hat{\mathbf{k}}(\beta)}=\sum_{\overline{\mathbf{a}} \in \overline{\mathcal{M}}} f_{\hat{\mathbf{a}}} \sum_{\beta: \overline{\mathbf{k}}(\beta)=\overline{\mathbf{a}}} \bar{\delta}_{\beta} \bar{v}_{\beta} y_{\hat{\mathbf{h}}(\beta)} .
$$

Copyright (c) by SIAM. Unauthorized reproduction of this article is prohibited. 
Thus,

$$
\sum_{\overline{\mathbf{a}} \in \overline{\mathcal{M}}} y_{\hat{\mathbf{a}}} \sum_{\beta: \overline{\mathbf{h}}(\beta)=\overline{\mathbf{a}}} \bar{\delta}_{\beta} \bar{v}_{\beta}\left(f_{\hat{\mathbf{k}}(\beta)}-f_{\hat{\mathbf{a}}}\right)=\sum_{\overline{\mathbf{a}} \in \overline{\mathcal{M}}} f_{\hat{\mathbf{a}}} \sum_{\beta: \overline{\mathbf{k}}(\beta)=\overline{\mathbf{a}}} \bar{\delta}_{\beta} \bar{v}_{\beta}\left(y_{\hat{\mathbf{h}}(\beta)}-y_{\hat{\mathbf{a}}}\right) .
$$

The above expression is equal to 0 for all periodic $f_{\hat{a}}$ if and only if

$$
\sum_{\beta: \overline{\mathbf{k}}(\beta)=\overline{\mathbf{a}}} \bar{\delta}_{\beta} \bar{v}_{\beta}\left(y_{\hat{\mathbf{h}}(\beta)}-y_{\hat{\mathbf{a}}}\right)=0 \text { for all } \overline{\mathbf{a}} \in \overline{\mathcal{N}} \text {. }
$$

A simple calculation shows that $y_{\hat{\mathbf{b}}}=y_{\hat{\mathbf{a}}}$ for all $\hat{\mathbf{a}}, \hat{\mathbf{b}} \in \overline{\mathcal{N}} / \varepsilon$ (if $\bar{v}_{e} \neq 0$ for all edges $e$. This is a generic condition that we assume is satisfied).

If we multiply the left-hand side of (A.29) by $y_{\hat{\mathbf{a}}}$ and add over all $\mathbf{a} \in \overline{\mathcal{M}}$, we obtain the expression in (A.31). Thus, the above discussion, the Fredholm alternative theory, and simple manipulations imply that there exists a solution $f_{\hat{\mathbf{a}}}$ (of (A.29)) that satisfies $f_{\hat{\mathbf{a}}+(n \overline{\mathbf{w}}+m \overline{\mathbf{z}}) / \varepsilon}=f_{\hat{\mathbf{a}}}$ for all integers $n$ and $m$ if and only if

$$
\frac{\partial \rho}{\partial \bar{t}}+\overline{\mathbf{V}} \cdot \bar{\nabla} \rho=0
$$

where $\bar{\nabla} \rho$ is the gradient of $\rho$ with respect to $\overline{\mathbf{x}}$.

A.3.3. Further expansions. Next we take derivatives of (A.33) with respect to $\bar{x}_{i}$ to obtain

$$
\frac{\partial^{2} \rho}{\partial \bar{t} \partial \bar{x}_{i}}=-\sum_{j}[\overline{\mathbf{V}}]_{j} \frac{\partial^{2} \rho}{\partial \bar{x}_{i} \partial \bar{x}_{j}}
$$

Analogously, taking derivatives of (A.33) with respect to $\bar{t}$ and using (A.34), we obtain

$$
\frac{\partial^{2} \rho}{\partial \bar{t}^{2}}=-\sum_{i}\left[\overline{\mathbf{V}}^{\prime}\right]_{i} \frac{\partial \rho}{\partial \bar{x}_{i}}-\sum_{i}[\overline{\mathbf{V}}]_{i} \frac{\partial^{2} \rho}{\partial \bar{x}_{i} \partial \bar{t}}=-\sum_{i}\left[\overline{\mathbf{V}}^{\prime}\right]_{i} \frac{\partial \rho}{\partial \bar{x}_{i}}+\sum_{i, j}[\overline{\mathbf{V}}]_{i}[\overline{\mathbf{V}}]_{j} \frac{\partial^{2} \rho}{\partial \bar{x}_{i} \partial \bar{x}_{j}}
$$

Using the last three identities, (A.28) becomes

$$
\begin{array}{r}
\bar{u}_{\overline{\mathbf{k}}(\beta)}\left(\bar{t}-\Delta \bar{t}_{\beta}\right)=\rho+\varepsilon\left\{\sum_{i} \frac{\partial \rho}{\partial \bar{x}_{i}}\left[\hat{\mathbf{k}}(\beta)-\hat{\mathbf{x}}+\frac{\bar{\ell}_{\beta}}{\bar{v}_{\beta}} \overline{\mathbf{V}}\right]_{i}+f_{\hat{\mathbf{k}}(\beta)}\right\} \\
+\varepsilon^{2}\left\{\frac{1}{2} \sum_{i, j} \frac{\partial^{2} \rho}{\partial \bar{x}_{i} \partial \bar{x}_{j}}\left[\hat{\mathbf{k}}(\beta)-\hat{\mathbf{x}}+\frac{\bar{\ell}_{\beta}}{\bar{v}_{\beta}} \overline{\mathbf{V}}\right]_{i}\left[\hat{\mathbf{k}}(\beta)-\hat{\mathbf{x}}+\frac{\bar{\ell}_{\beta}}{\bar{v}_{\beta}} \overline{\mathbf{V}}\right]_{j}\right. \\
\left.-\frac{1}{2} \frac{\bar{\ell}_{\beta}}{\bar{v}_{\beta}} \sum_{i}\left(\frac{\bar{\ell}_{\beta}}{\bar{v}_{\beta}}[\overline{\mathbf{V}}]_{i}\right)^{\prime} \frac{\partial \rho}{\partial \bar{x}_{i}}-\frac{\partial \rho}{\partial \tau} \frac{\bar{\ell}_{\beta}}{\bar{v}_{\beta}}+\sum_{i} \frac{\partial f_{\hat{\mathbf{k}}(\beta)}}{\partial \bar{x}_{i}}[\hat{\mathbf{k}}(\beta)-\hat{\mathbf{x}}]_{i}-\frac{\partial f_{\hat{\mathbf{k}}(\beta)}}{\partial \bar{t}} \frac{\bar{\ell}_{\beta}}{\bar{v}_{\beta}}+g_{\hat{\mathbf{k}}(\beta)}\right\} .
\end{array}
$$

We now plug into (A.21) the expressions for $\bar{u}_{\hat{\mathbf{k}}(\beta)}\left(\bar{t}-\Delta \bar{t}_{\beta}\right)$ and $\bar{u}_{a}(\bar{t})$ given by (A.36) and (A.25), respectively, to obtain, after some algebraic manipulations and 
making use of (A.29), that

$$
\begin{array}{r}
\left(\sum_{\{\beta: \overline{\mathbf{h}}(\beta)=\overline{\mathbf{a}}\}} \bar{\delta}_{\beta} \bar{\ell}_{\beta}\right) \frac{\partial \rho}{\partial \tau}+\sum_{\{\beta: \overline{\mathbf{h}}(\beta)=\overline{\mathbf{a}}\}}\left[\bar{\delta}_{\beta} \bar{v}_{\beta}\left(\bar{\nabla} f_{\hat{\mathbf{a}}} \cdot(\hat{\mathbf{a}}-\hat{\mathbf{x}})-\bar{\nabla} f_{\hat{\mathbf{k}}(\beta)} \cdot(\hat{\mathbf{k}}(\beta)-\hat{\mathbf{x}})\right)\right. \\
\left.+\frac{\bar{\delta}_{\beta}}{2} \bar{\ell}_{\beta}^{2}\left(\frac{\overline{\mathbf{V}}}{\bar{v}_{\beta}}\right)^{\prime} \cdot \bar{\nabla} \rho+\bar{\delta}_{\beta} \bar{\ell}_{\beta} \frac{\partial f_{\hat{\mathbf{k}}(\beta)}}{\partial \bar{t}}\right] \\
=\frac{1}{2} \sum_{\{\beta: \overline{\mathbf{h}}(\beta)=\overline{\mathbf{a}}\}} \bar{\delta}_{\beta} \bar{v}_{\beta} \sum_{i, j} \frac{\partial^{2} \rho}{\partial \bar{x}_{i} \partial \bar{x}_{j}}\left(\left[\hat{\mathbf{k}}(\beta)-\hat{\mathbf{a}}+\frac{\bar{\ell}_{\beta}}{\bar{v}_{\beta}} \overline{\mathbf{V}}\right]_{i}\left[\hat{\mathbf{k}}(\beta)-\hat{\mathbf{a}}+\frac{\bar{\ell}_{\beta}}{\bar{v}_{\beta}} \overline{\mathbf{V}}\right]_{j}\right. \\
\left.2+[\hat{\mathbf{a}}-\hat{\mathbf{x}}]_{i}\left[\hat{\mathbf{k}}(\beta)-\hat{\mathbf{a}}+\frac{\bar{\ell}_{\beta}}{\bar{v}_{\beta}} \overline{\mathbf{V}}\right]_{j}\right)+\sum_{\{\beta: \overline{\mathbf{h}}(\beta)=\overline{\mathbf{a}}\}} \bar{\delta}_{\beta} \bar{v}_{\beta}\left(g_{\hat{\mathbf{k}}(\beta)}-g_{\hat{\mathbf{a}}}\right) .
\end{array}
$$

Taking the gradient of (A.29), taking the dot product of the result with ( $\hat{\mathbf{a}}-$ $\hat{\mathbf{x}}$ ), making use of previous equations, performing some algebraic manipulations, and noting that $\bar{v}_{\beta}(\hat{\mathbf{a}}-\hat{\mathbf{k}}(\beta))=\bar{\ell}_{\beta} \overline{\mathbf{v}}_{\beta}$, (A.37) can be reduced to

$$
\begin{aligned}
& \left(\sum_{\{\beta: \overline{\mathbf{h}}(\beta)=\overline{\mathbf{a}}\}} \bar{\delta}_{\beta} \bar{\ell}_{\beta}\right) \frac{\partial \rho}{\partial \tau}+\sum_{\{\beta: \overline{\mathbf{h}}(\beta)=\overline{\mathbf{a}}\}} \bar{\delta}_{\beta} \bar{\ell}_{\beta}\left[\bar{\nabla} f_{\hat{\mathbf{k}}(\beta)} \cdot \overline{\mathbf{v}}_{\beta}+\frac{\bar{\ell}_{\beta}}{2}\left(\frac{\overline{\mathbf{V}}}{\bar{v}_{\beta}}\right)^{\prime} \cdot \bar{\nabla} \rho+\frac{\partial f_{\hat{\mathbf{k}}(\beta)}}{\partial \bar{t}}\right] \\
= & \sum_{\{\beta: \overline{\mathbf{h}}(\beta)=\overline{\mathbf{a}}\}} \bar{\delta}_{\beta} \bar{v}_{\beta}\left(g_{\hat{\mathbf{k}}(\beta)}-g_{\hat{\mathbf{a}}}\right)+\frac{1}{2} \sum_{i, j} \frac{\partial^{2} \rho}{\partial \bar{x}_{i} \partial \bar{x}_{j}} \sum_{\{\beta: \overline{\mathbf{h}}(\beta)=\overline{\mathbf{a}}\}} \frac{\bar{\delta}_{\beta} \bar{\ell}_{\beta}^{2}}{\bar{v}_{\beta}}\left[\overline{\mathbf{v}}_{\beta}-\overline{\mathbf{V}}\right]_{i}\left[\overline{\mathbf{v}}_{\beta}-\overline{\mathbf{V}}\right]_{j} .
\end{aligned}
$$

A.4. Fredholm alternative. Long time equation for $\rho$. Dispersion tensor. Following the same arguments to obtain (A.33), we have that there exists a solution $g_{\hat{\mathbf{a}}}$ of (A.38) that is periodic in $\hat{\mathbf{a}}$ if and only if

$$
\begin{array}{r}
\sum_{\overline{\mathbf{a}} \in \overline{\mathcal{M}}}\left\{\left(\sum_{\{\beta: \overline{\mathbf{k}}(\beta)=\overline{\mathbf{a}}\}} \bar{\delta}_{\beta} \bar{\ell}_{\beta}\right) \frac{\partial f_{\hat{\mathbf{a}}}}{\partial \bar{t}}+\left(\sum_{\{\beta: \overline{\mathbf{k}}(\beta)=\overline{\mathbf{a}}\}} \bar{\delta}_{\beta} \bar{\ell}_{\beta} \overline{\mathbf{v}}_{\beta}\right) \cdot \bar{\nabla} f_{\hat{\mathbf{a}}}\right\} \\
+\sum_{\overline{\mathbf{a}} \in \overline{\mathcal{M}}}\left\{\left(\sum_{\{\beta: \overline{\mathbf{k}}(\beta)=\overline{\mathbf{a}}\}} \bar{\delta}_{\beta} \bar{\ell}_{\beta}\right) \frac{\partial \rho}{\partial \tau}+\left(\sum_{\{\beta: \overline{\mathbf{k}}(\beta)=\overline{\mathbf{a}}\}} \frac{\bar{\delta}_{\beta}}{2} \bar{\ell}_{\beta}^{2}\left(\frac{\overline{\mathbf{V}}}{\bar{v}_{\beta}}\right)^{\prime}\right) \cdot \bar{\nabla} \rho\right\} \\
=\frac{1}{2} \sum_{i, j} \frac{\partial^{2} \rho}{\partial \bar{x}_{i} \partial \bar{x}_{j}} \sum_{\overline{\mathbf{a}} \in \overline{\mathcal{M}}\{\beta: \overline{\mathbf{k}}(\beta)=\overline{\mathbf{a}}\}} \sum_{\bar{\delta}_{\beta} \bar{\ell}_{\beta}^{2}}\left[\bar{v}_{\beta}-\overline{\mathbf{V}}\right]_{i}\left[\overline{\mathbf{v}}_{\beta}-\overline{\mathbf{V}}\right]_{j} .
\end{array}
$$

Let $\mathbf{F}_{\hat{\mathbf{a}}}=\mathbf{F}_{\hat{\mathbf{a}}}(\bar{t})$ be a solution of

$$
\sum_{\{\beta: \overline{\mathbf{h}}(\beta)=\overline{\mathbf{a}}\}} \bar{\delta}_{\beta} \bar{v}_{\beta}\left(\mathbf{F}_{\hat{\mathbf{k}}(\beta)}-\mathbf{F}_{\hat{\mathbf{a}}}\right)=\sum_{\{\beta: \overline{\mathbf{h}}(\beta)=\overline{\mathbf{a}}\}} \bar{\delta}_{\beta} \bar{\ell}_{\beta}\left(\overline{\mathbf{v}}_{\beta}-\overline{\mathbf{V}}\right)
$$

that is periodic in $\bar{t}$ and $\hat{\mathbf{a}}$, i.e., $\mathbf{F}_{\hat{\mathbf{a}}+(n \overline{\mathbf{w}}+m \overline{\mathbf{q}}) / \varepsilon}(\bar{t}+p)=\mathbf{F}_{\hat{\mathbf{a}}}(\bar{t})$ for all integers $n, m, p$. A simple calculation shows that $f_{\hat{\mathbf{a}}}$ is a periodic (in $\hat{\mathbf{a}}$ and $\bar{t}$ ) solution of (A.29) if and only if

$$
f_{\hat{\mathbf{a}}}(\overline{\mathbf{x}}, \bar{t}, \tau)=\mathbf{F}_{\hat{\mathbf{a}}}(\bar{t}) \cdot \bar{\nabla} \rho(\bar{x}, \bar{t}, \tau)+\psi(\bar{x}, \bar{t}, \tau),
$$

Copyright (c) by SIAM. Unauthorized reproduction of this article is prohibited. 
where $\psi$ is an arbitrary function that is periodic on $\bar{t}$.

Note that $\{\beta: \overline{\mathbf{k}}(\beta)=\overline{\mathbf{a}}$ and $\overline{\mathbf{a}} \in \overline{\mathcal{M}}\}$ is a set that contains exactly one edge per equivalent class. Thus, the sums in equation (A.39) are spatial averages, i.e., $\sum_{\overline{\mathbf{a}} \in \overline{\mathcal{M}}} \sum_{\{\beta: \overline{\mathbf{k}}(\beta)=\overline{\mathbf{a}}\}}=\sum_{\beta \in \mathcal{F}}$, where we recall that $\mathcal{F}$ is any set of edges that contains exactly one edge per equivalent class. Using this observation, (A.41), and some simple algebraic manipulations, we transform (A.39) in

$$
\begin{aligned}
& \left(\sum_{\beta \in \mathcal{F}} \bar{\delta}_{\beta} \bar{\ell}_{\beta}\right)\left(\frac{\partial \rho}{\partial \tau}+\frac{\partial \psi}{\partial \bar{t}}+\overline{\mathbf{V}} \cdot \bar{\nabla} \psi\right)+\left(\sum_{\beta \in \mathcal{F}} \bar{\delta}_{\beta} \bar{\ell}_{\beta} \mathbf{F}_{\hat{\mathbf{k}}(\beta)}^{\prime}+\frac{\bar{\delta}_{\beta}}{2} \bar{\ell}_{\beta}^{2}\left(\frac{\overline{\mathbf{V}}}{\bar{v}_{\beta}}\right)^{\prime}\right) \cdot \bar{\nabla} \rho \\
= & \frac{1}{2} \sum_{i, j} \frac{\partial^{2} \rho}{\partial \bar{x}_{i} \partial \bar{x}_{j}} \sum_{\beta \in \mathcal{F}} \bar{\delta}_{\beta} \bar{\ell}_{\beta}\left(\frac{\bar{\ell}_{\beta}}{\bar{v}_{\beta}}\left[\overline{\mathbf{v}}_{\beta}-\overline{\mathbf{V}}\right]_{i}\left[\overline{\mathbf{v}}_{\beta}-\overline{\mathbf{V}}\right]_{j}+2\left[\mathbf{F}_{\hat{\mathbf{k}}(\beta)}\right]_{i}\left[\overline{\mathbf{V}}-\overline{\mathbf{v}}_{\beta}\right]_{j}\right) .
\end{aligned}
$$

We now make the change of variables

$$
\overline{\mathbf{y}}=\overline{\mathbf{x}}-\int_{0}^{\bar{t}} \overline{\mathbf{V}}(s) \mathrm{d} s .
$$

To avoid confusion, we denote $\rho$ by $\bar{\rho}$ when the new independent variables $(\overline{\mathbf{y}}, \bar{t}, \tau)$ are used. In these new variables, (A.33) becomes $\partial \bar{\rho} /(\partial \bar{t})=0$, from which we get that $\bar{\rho}$ is a function that depends only on $\overline{\mathbf{y}}$ and $\tau$, i.e., $\bar{\rho}=\bar{\rho}(\overline{\mathbf{y}}, \tau)$.

Analogously, $\bar{\psi}$ is simply $\psi$, but only when the new independent variables $(\overline{\mathbf{y}}, \bar{t}, \tau)$ are used. The changes that occur in (A.42) when the new variables are used are the following: (1) The term $\overline{\mathbf{V}} \cdot \bar{\nabla} \psi$ is removed; (2) derivatives with respect to $\overline{\mathbf{x}}$ are replaced by derivatives with respect to $\overline{\mathbf{y}}$; and (3) $\rho$ is replaced by $\bar{\rho}$ and $\psi$ by $\bar{\psi}$. After making this change of variables, we integrate the resulting equation (A.42) with respect to $\bar{t}$ over a period, from $\bar{t}=\bar{t}_{0}$ to $\bar{t}=\bar{t}_{0}+1$, and divide by $\sum_{\beta \in \mathcal{F}} \bar{\delta}_{\beta} \bar{\ell}_{\beta}$ to obtain

$$
\frac{\partial \bar{\rho}}{\partial \tau}(\overline{\mathbf{y}}, \tau)+\bar{\psi}\left(\overline{\mathbf{y}}, \bar{t}_{0}+1, \tau\right)-\bar{\psi}\left(\overline{\mathbf{y}}, \bar{t}_{0}, \tau\right)=\sum_{i, j} \bar{D}_{i j} \frac{\partial^{2} \bar{\rho}}{\partial y_{i} \partial y_{j}}(\overline{\mathbf{y}}, \tau)
$$

where

$$
\begin{array}{r}
\bar{D}_{i j}=\frac{1}{2 \sum_{\beta \in \mathcal{F}} \bar{\delta}_{\beta} \bar{\ell}_{\beta}} \sum_{\beta \in \mathcal{F}} \bar{\delta}_{\beta} \bar{\ell}_{\beta} \int_{0}^{1}\left(\frac{\bar{\ell}_{\beta}}{\bar{v}_{\beta}}\left[\overline{\mathbf{v}}_{\beta}-\overline{\mathbf{V}}\right]_{i}\left[\overline{\mathbf{v}}_{\beta}-\overline{\mathbf{V}}\right]_{j}\right. \\
\left.+\left[\mathbf{F}_{\hat{\mathbf{k}}(\beta)}\right]_{i}\left[\overline{\mathbf{V}}-\overline{\mathbf{v}}_{\beta}\right]_{j}+\left[\mathbf{F}_{\hat{\mathbf{k}}(\beta)}\right]_{j}\left[\overline{\mathbf{V}}-\overline{\mathbf{v}}_{\beta}\right]_{i}\right) \mathrm{d} \bar{t}
\end{array}
$$

Finally, the dependence of $\bar{\rho}$ in $\tau$ is obtained by requiring $\bar{\psi}$ to be bounded. Given (A.44), we note that this occurs only if

$$
\frac{\partial \bar{\rho}}{\partial \tau}(y, \tau)=\sum_{i, j} \bar{D}_{i j} \frac{\partial^{2} \bar{\rho}}{\partial y_{i} \partial y_{j}}(y, \tau) .
$$

The result stated in section 3 is obtain by going back to the original dimensional variables. 
[1] P. M. Adler And H. Brenner, Transport processes in spatially periodic capillary networks-II. Taylor dispersion with mixing vertices, PhysicoChem. Hydrodyn., 5 (1984), pp. 269-285.

[2] P. M. Adler, Porous Media. Geometry and Transports, Butterworth-Heinemann, Stoneham, MA, 1992.

[3] R. ARIS, On the dispersion of solute matter in a fluid flowing through a tube, Proc. Roy. Soc. London Ser. A, 235 (1956), pp. 67-77.

[4] G. K. Batchelor, An Introduction to Fluid Dynamics, Cambridge University Press, New York, 1973.

[5] J. BEAR, On the tensor form of dispersion in porous media, J. Geophys. Res., 66 (1961), pp. 1185-1197.

[6] J. Bear, Dynamics of Fluids in Porous Media, Dover Publications, New York, 1972.

[7] Berkowitz And Balberg, Percolation theory and its applications to ground water hydrology, Water Resour. Res., 29 (1993), pp. 775-794.

[8] B. Berkowitz And R. P. Ewing, Percolation theory and network modeling applications in soil physics, Surveys in Geophysics, 19 (1998), pp. 23-72.

[9] B. Berkowitz, Dispersion in Heterogeneous Geological Formations, Kluwer Academic Publishers, London, 2001.

[10] B. Berkowitz, Characterizing flow and transport in fractured geological media: A review, in Adv. Water Res., 25 (2002), pp. 861-884.

[11] H. Brenner, Dispersion resulting from flow through spatially periodic porous media, Philos. Trans. Roy. Soc. London Ser. A, 297 (1980), pp. 81-133.

[12] H. Brenner, A general theory of Taylor dispersion phenomena, PhysicoChem. Hydrodyn., 1 (1980), pp. 91-123.

[13] H. Brenner And P. M. Adler, Transport Processes in Porous Media, Hemisphere/McGrawHill, New York, 1985.

[14] H. Brenner and D. A. Edwards, Macrotransport Processes, Butterworth-Heinemann Seri. Chem. Engrg., Butterworth-Heinemann, Boston, MA, 1993.

[15] C. BRuderer AND Y. Bernabe, Network modeling of dispersion: Transition from Taylor dispersion in homogeneous networks to mechanical dispersion in very heterogeneous ones, Water Resour. Res., 37 (2001), pp. 897-908.

[16] C. Bruderer-Wang, P. Cowie, Y. Bernabe, and I. Main, Relating flow channeling to tracer dispersion in heterogeneous networks, Adv. in Water Res., 27 (2004), pp. 843-855.

[17] M. L. Brusseau, Transport of reactive contaminants in heterogeneous porous media, Rev. Geophys., 32 (1994), pp. 285-313.

[18] R. P. CARBonell, Effect of pore distribution and flow segregation on dispersion in porous media, Chem. Eng. Sci., 34 (1979), pp. 1031-1039.

[19] H. R. Cedergren, Seepage, Drainage, and Flow Nets, John Wiley, New York, 1989.

[20] E. Charlaix and H. Gayvallet, Hydrodynamic dispersion in networks of capillaries of random permeability, Europhys. Lett., 16 (1991), pp. 259-264.

[21] K. H. Conts, Dead-end pore volume and dispersion in porous media, Soc. Pet. Eng. J., 4 (1964), pp. 73-84.

[22] J. H. Cushman, Dynamics of Fluids in Hierarchical Porous Media, Academic Press, New York, 1990.

[23] J. H. Cushman, L. S. Bennethum, and B. X. Hu, A primer on upscaling tools for porous media, Adv. in Water Res., 25 (2002), pp. 1043-1067.

[24] G. DAGAn, Theory of solute transport by groundwater, in Ann. Rev. Fluid Mech., 19 (1987), pp. $183-215$.

[25] G. Dagan, Flow and Transport in Porous Formations, Springer-Verlag, New York, 1989.

[26] G. DaGan, Transport in heterogeneous porous formations: Spatial moments, ergodicity, and effective dispersion, Water Resour. Res., 26 (1990), pp. 1281-1290.

[27] L. De Arcangelis, J. Koplik, S. Redner, and D. Wilkinson, Hydrodynamic dispersion in network models of porous media, Phys. Rev. Lett., 57 (1986), pp. 996-999.

[28] S. Didierjean, H. P. Amaral Souto, R. Delannay, and C. Moyne, Dispersion in periodic porous media. Experience versus theory for two-dimensional systems, Chem. Eng. Sci., 52 (1997), pp. 1861-1874.

[29] K. D. Dorfman and H. Brenner, Generalized Taylor-Aris dispersion in discrete spatially periodic networks: Microfluidic applications, Phys. Rev. E, 65 (2002), 021103.

[30] F. A. L. Dullien, Porous Media. Fluid Transport and Pore Structure, 2nd ed., Academic Press, New York, 1992.

Copyright (c) by SIAM. Unauthorized reproduction of this article is prohibited. 
[31] D. A. Edwards, M. Shapiro, H. Brenner, And M. Shapira, Dispersion of inert solutes in spatially periodic two-dimensional porous media, Transp. Porous Media, 6 (1991), pp. 337-358.

[32] A. M. M. Elfeki, G. J. M. Uffink, And F. B. J. Barends, Groundwater Contaminant Transport. Impact of Heterogeneous Characterization: A New View on Dispersion, A. A. Balkema, Rotterdam, The Netherlands, 1997.

[33] I. FATT, The network model of porous media-I. Capillary pressure characteristics, Trans. Am. Inst. Min. Engrs., 207 (1956), pp. 144-159.

[34] I. FATT, The network model of porous media-II. Dynamic properties of a single size tube network, Trans. Am. Inst. Min. Engrs., 207 (1956), pp. 160-163.

[35] I. FATT, The network model of porous media-III. Dynamic properties of networks with tube radius distributions, Trans. Am. Inst. Min. Engrs., 207 (1956), pp. 164-181.

[36] J. Feyen, D. Jacques, A. Timmerman, and J. Vanderborght, Modeling water flow and solute transport in heterogeneous soils: A review of recent approaches, J. Agric. Engng. Res., 70 (1998), pp. 231-256.

[37] J. J. Fried and M. A. Combarnous, Dispersion in porous media, Adv. Hydrosci., 7 (1971), pp. 169-282.

[38] G. R. Gavalas and S. Kim, Periodic capillaries models of diffusion in porous solids, Chem. Eng. Sci., 36 (1981), pp. 1111-1122.

[39] G. H. Goldsztein, Transport of nutrients in bones, SIAM J. Appl. Math., 65 (2005), pp. $2128-2140$.

[40] D. J. Gunn and C. Pryce, Dispersion in packed beds, Trans. Inst. Chem. Eng., 47 (1969), pp. T342-T350.

[41] F. J. M. HoRn, Calculation of dispersion coefficients by means of moments, AIChE J., 17 (1971), pp. 613-620.

[42] U. Hornung, Miscible displacement, in Homogenization and Porous Media, U. Hornung, ed., Springer, New York, 1997.

[43] M. L. Knothe Tate and U. Knothe, An ex vivo model to study the transport processes and fluid flow in loaded bone, J. Biomech., 33 (2000), pp. 247-254.

[44] M. L. Knothe Tate, P. Niederer, and U. Knothe, In vivo tracer transport through the lacunocanalicular system of rat bone in an environment devoid of mechanical loading, Bone, 22 (1998), pp. 107-117.

[45] M. L. Knothe Tate, U. Knothe, and P. Niederer, Experimental elucidation of mechanical load-induced fluid flow and its potential role in bone metabolism and functional adaptation, Am. J. Med. Sci., 316 (1998), pp. 189-195.

[46] D. L. Koch and J. F. Brady, Dispersion in fixed beds, J. Fluid Mech., 154 (1985), pp. 399-427.

[47] S. Koplik, S. ReDNeR, AND D. Wilkinson, Transport and dispersion in random networks with percolation disorder, Phys. Rev. A (3), 37 (1988), pp. 2619-2636.

[48] R. MAURI, Dispersion, convection and reaction in porous media, Phys. Fluids A, 3 (1991), pp. $743-756$.

[49] C. C. MeI, Method of homogenization applied to dispersion in porous media, Transp. in Porous Media, 9 (1992), pp. 261-274.

[50] K. Piekarski And M. Munro, Transport mechanism operating between blood supply and osteocytes in long bones, Nature, 269 (1977), pp. 80-82.

[51] J. Rubinstein AND R. MAuRI, Dispersion and convection in periodic porous media, SIAM J. Appl. Math., 46 (1986), pp. 1018-1023.

[52] P. G. Saffman, Dispersion flow through a network of capillaries, Chem. Eng. Sci., 11 (1959), pp. $125-129$.

[53] P. G. Saffman, A theory of dispersion in a porous medium, J. Fluid Mech., 6 (1959), pp. 321-349.

[54] P. G. Saffman, Dispersion due to molecular diffusion and macroscopic mixing in flow through a network of capillaries, J. Fluid Mech., 7 (1960), pp. 194-208.

[55] M. Sahimi, A. A. Heiba, B. D. Hughes, H. T. Davis, and L. E. Scriven, Dispersion in Flow through Porous Media, SPE 10969, Soc. Petrol. Engrs., New Orleans, LA, 1982.

[56] M. Sahimi, B. D. Hughes, L. E. Scriven, and H. T. Davis, Stochastic transport in disordered systems, J. Chem. Phys., 78 (1983), pp. 6849-6864.

[57] M. Sahimi, H. T. Davis, and L. E. Scriven, Dispersion in disordered porous media, Chem. Eng. Commun., 23 (1983), pp. 329-341.

[58] SAнimi, Flow and Transport in Porous Media and Fractured Rock, John Wiley, New York, 1995.

[59] J. Salles, J.-F. Thovert, R. Delannay, L. Prevors, J.-L. Auriault, and P. M. Adler, Taylor dispersion in porous media. Determination of the dispersion tensor, Phys. Fluids A, 5 (1993), pp. 2348-2376.

Copyright $@$ by SIAM. Unauthorized reproduction of this article is prohibited. 
[60] M. SARdin AND D. Schweich, Modeling the nonequilibrium transport of linearly interacting solutes in porous media: A review, Water Resourc. Res. 27 (1991), pp. 2287-2307.

[61] A. E. Scheidegger, The Physics of Flow through Porous Media, 2nd ed., Toronto University Press, Toronto, 1963.

[62] V. I. Selyakov and V. V. Kadet, Percolation Models for Transport in Porous Media, Kluwer Academic Publishers, Boston, 1996.

[63] G. I. TAYLOR, Dispersion of soluble matter in solvent flowing slowly through a tube, Proc. Roy. Soc. London Ser. A, 223 (1953), pp. 446-468.

[64] S. W. Wheatcraft, G. A. Sharp, And S. W. Tyler, Fluid flow and solute transport in fractal heterogeneous porous media, in Dynamics of Fluid in Hierarchical Porous Media, J. H. Cushman, ed., Academic Press, New York, 1990, pp. 305-326.

[65] L. Wang, S. C. Cowin, S. Weinbaum, and S. P. Fritton, Modeling tracer transport in an osteon under cyclic loading, Ann. Biomed. Eng., 28 (2000), pp. 1200-1209.

Copyright $@$ by SIAM. Unauthorized reproduction of this article is prohibited. 